\title{
Association of Mean Platelet Volume with Angiographic Thrombus Burden in Patient with ST-Segment Elevation Myocardial Infarction Undergoing Primary Percutaneous Coronary Intervention
}

\author{
Mamdouh Helmy El Tahan', MD; Attia Morsy Shokr¹, MD; Waleed Sharaf Mehana ${ }^{1 *}$, M.B.B.Ch
}

\author{
*Corresponding author: \\ Waleed Sharaf Mehana \\ (+20) 1004342584 \\ waleadmehana@gmail.com
}

Received for publication April 13, 2020;

Accepted May 10, 2020; Published

online June 6, 2020.

\begin{abstract}
Copyright 2020 The Authors published by Al-Azhar University, Faculty of Medicine, Cairo, Egypt. All rights reserved. This an open-access article distributed under the legal terms, where it is permissible to download and share the work provided it is properly cited. The work cannot be changed in any way or used commercially.
\end{abstract}

\section{doi: 10.21608/aimj.2020.94239}

${ }^{1}$ Department of Cardiology, Faculty of Medicine, Al-Azhar University, Cairo, Egypt.

Disclosure: The authors have no financial interest to declare in relation to the content of this article. The article processing charge was paid for by the authors.

Authorship: All authors have a substantial contribution to the article.

\begin{abstract}
Background: ST-elevation myocardial infarction (STEMI) is one of the leading causes of mortality and morbidity worldwide. However, survival after acute STEMI has considerably improved due to increasing symptom recognition, accurate diagnosis, and effective timely reperfusion.
\end{abstract}

Objectives: To assess the association of mean platelet volume (MPV) as a biomarker for angiographic thrombus burden in a patient with ST-segment elevation myocardial infarction undergoing primary percutaneous coronary intervention (PPCI).

Patient and methods: This study was conducted on 125 patients admitted to ElMokatem health insurance hospital, with the diagnosis of acute ST-segment elevation myocardial infarction (STEMI) treated with PPCI in the period between December 2018 and October 2019.

Results: Thrombus burden level determined by TIMI thrombus grade (TIMITG) was significantly higher in the high MPV group (p-value $<0.001)$. The LTB group had significantly higher admission MPV compared with the STB group (10.42 \pm 0.79 vs. $8.88 \pm 0.84, \mathrm{P}<0.001)$. In a receiver operating characteristics analysis, MPV > 9.75 predicted LTB with $83.3 \%$ sensitivity and $86.2 \%$ specificity. Multivariate logistic regression analysis demonstrated MPV was an independent predictor of large intracoronary thrombus burden in patients with STEMI undergoing PPCI

Conclusion: In STEMI patients who underwent PPCI, MPV on admission was significantly higher in those who experienced a high thrombus burden. MPV on admission may serve as a readily available an independent predictor of the thrombus burden in STEMI patients undergoing PPCI.

Keywords: Mean Platelet volume, TIMITG, HTB, PCI, PPCI, STEMI

\section{INTRODUCTION}

Despite the improvement in the management of intracoronary thrombus, including intracoronary administration of glycoprotein IIb/IIIa inhibitors, thrombus aspiration and thrombectomy, PPCI for STEMI patients with large intracoronary thrombus burden still carries an increased risk of complications for patients ${ }^{\mathbf{1}}$.

Because large intracoronary thrombus possesses prognosis importance, it requires early recognition and effective treatment. Thus, establishing preprocedural factors predisposing for thrombus burden is in demand, which may improve risk stratification and provide therapeutic implications in the clinical evaluation of patients with STEMI before $\mathrm{PPCI}^{2}$.

Platelets play an important role in cardiovascular disease both in the pathogenesis of atherosclerosis and in the development of acute thrombotic events. Mediators of inflammation, atherosclerosis, coagulation, and thrombosis can be released by platelets. Platelets vary in size and are hemostatic in nature. The larger the platelets in the circulation, the more granules they contain and release higher amounts of prothrombotic and vasoactive factors and undergo rapid aggregation under agonist stimulation ${ }^{3}$.

Based on their thrombotic potential, platelets have shown a substantial contribution to the pathogenesis of STEMI. Mean platelet volume; a measure of the platelet size has been shown to correlate with platelets' reactivity ${ }^{4}$.

Coronary artery thrombosis is the final pathogenic mechanism of acute ischemic events, including myocardial infarction and sudden cardiac arrest. There are complex interactions among the atherosclerotic artery, endothelial injury and dysfunction, vasospasm, and platelet activation. Plaque rupture exposes thrombogenic 
subendothelial components, leading to platelet deposition and activation ${ }^{5}$

\section{PATIENT AND METHODS}

\section{Patient:}

This study was conducted on 125 patients admitted to ElMokatem health insurance hospital, with the diagnosis of acute ST-segment elevation myocardial infarction (STEMI) treated with primary percutaneous coronary intervention in the period between December 2018 and October 2019. Inclusion criteria were continuous chest pain for at least 30 minutes within the preceding 12 hours; ECG showing ST-segment elevation $1 \mathrm{~mm}$ in two contiguous limb leads or $2 \mathrm{~mm}$ in two contiguous precordial leads; new-onset left bundle branch block; and age 18 years. Exclusion criteria were hematologic disorders; and patients with missing admission MPV data and/or follow-up information.

\section{Laboratory analysis and echocardiography:}

In all cases, venous peripheral blood samples were drawn on admission, then collected into standardized tubes containing dipotassium ethylenedinitrotetraacetic acid (EDTA) reagent and stored at room temperature. Complete blood picture including the MPV measurements was performed within 30 minutes of admission. Echocardiography investigation was routinely performed to all patients within 48 hours after PPCI. Left ventricular ejection fraction (LVEF) is measured using both M-mode and $2 \mathrm{D}$ eyeballing.

\section{Coronary angiography and intervention:}

Coronary angiography in standardized projections and PPCI was performed for each case. Digital angiograms were then analyzed by an experienced interventional cardiologist who was blind to the MPV results. Intracoronary thrombus was graded as; TIMI thrombus Grade $0(\mathrm{G} 0)$, no cine angiographic characteristics of thrombus are present; TIMI thrombus Grade 1 (G1), a possible thrombus is present, with such angiography characteristics as reduced contrast density, haziness, irregular lesion contour, or a smooth convex "meniscus" at the site of total occlusion suggestive of, but not diagnostic of, thrombus; TIMI thrombus Grade 2 (G2), there is definite thrombus, with greatest dimensions $<1 / 2$ the vessel diameter; TIMI thrombus Grade 3 (G3), there is definite thrombus, but with greatest linear dimension $>1 / 2$ but $<2$ vessel diameters; TIMI thrombus Grade 4 (G4), there is definite thrombus, with the largest dimension $>2$ vessel diameters; TIMI thrombus Grade 5 (G5), there is total occlusion (unable to assess thrombus burden due to total vessel occlusion).
In TIMI thrombus Grade 5 (G5), we reclassified the thrombus into G0 to 4 after the wire passed the lesion or small size balloon $(<1.5 \mathrm{~mm})$ was dilated, and coronary flow was obtained ${ }^{6}$.

Patients were divided into two groups according to the final TIMI thrombus grade: low-thrombus burden group (LTB) with a grade of 0-3, and high-thrombus burden group (HTB) with a grade of $4 \& 5$.

\section{Statistical analysis:}

Recorded data were analyzed using the statistical package for social sciences, version 20.0 (SPSS Inc., Chicago, Illinois, USA). Quantitative data were expressed as mean \pm standard deviation (SD). Qualitative data were expressed as frequency and percentage. The differences between groups of continuous variables with a normal distribution (age, LVEF, stent parameters, and hematological parameters) were tested by independent samples t-test. Categorical variables were summarized as percentages and compared with the chi-square test. A univariate analysis was first performed to test for the association of the high-thrombus burden and several potentially impacting variables (age, sex, history of diabetes mellitus, HTN, LVEF, creatine level, and hemoglobin level). Multivariate logistic regression analysis was then used to identify independent predictors of high thrombus burden using variables (MPV, time from symptom onset to PPCI, and platelets count) that reached a trend-level effect $(\mathrm{p}<0.1)$ in the univariate analyses. The receiver operating characteristics (ROC) curve was used to determine the cut-off value of MPV to predict the high-thrombus burden. A two-sided p-value of $<0.05$ was considered significant.

\section{RESULTS}

During the study period, 125 patients were enrolled. There were 60 patients (48\%) in the HTB group (mean age 59.45 \pm 10.79 and $88.3 \%$ men), and 65 patients $(52 \%)$ in the LTB group (mean age $60.28 \pm 10.23$ and $86.2 \%$ men). Baseline clinical characteristics of the study population stratified by thrombus burden are presented in Table I. There is no significant differences were observed for other clinical variables between two groups. Baseline laboratory characteristics of the study population stratified by thrombus burden are summarized in Table II. The admission MPV was significantly higher in the HTB group than in the LTB group $(10.42 \pm 0.79$ vs. $8.88 \pm 0.84$, P < 0.001 ). The left anterior descending coronary artery was shown to be the most frequently involved IRA in HTB group compared with the LTB group (53.3\% vs. 56.9\%). Compared with patients with HTB, pre interventional TIMI < 3 flow was more in patients with HTB with Pvalue 0.021 . As anticipated, thrombus aspiration device was used more frequently in the HTB group versus LTB group (11.7\% vs. $1.5 \%, \mathrm{P} 0.028)$. 


\begin{tabular}{|c|c|c|c|c|c|c|}
\hline & & \multicolumn{4}{|c|}{ Thrombus burden } & \multirow{3}{*}{ P-valuc } \\
\hline & & \multicolumn{2}{|c|}{ LTB } & \multicolumn{2}{|c|}{ HTB } & \\
\hline & & Count & $\%$ & Count & $\%$ & \\
\hline \multirow{3}{*}{ Smoking } & YES & 32 & $49.2 \%$ & 30 & $50.0 \%$ & \multirow{3}{*}{0.501} \\
\hline & $\mathbf{X}$ & 7 & $10.8 \%$ & 3 & $5.0 \%$ & \\
\hline & NO & 26 & $40.0 \%$ & 27 & $45.0 \%$ & \\
\hline \multirow{2}{*}{ Family history } & YES & 11 & $16.9 \%$ & 7 & $11.7 \%$ & \multirow{2}{*}{0.403} \\
\hline & NO & 54 & $83.1 \%$ & 53 & $88.3 \%$ & \\
\hline \multirow{2}{*}{ DM } & YES & 29 & $44.6 \%$ & 29 & $48.3 \%$ & \multirow{2}{*}{0.677} \\
\hline & NO & 36 & $55.4 \%$ & 31 & $51.7 \%$ & \\
\hline \multirow{2}{*}{ HTN } & YES & 31 & $47.7 \%$ & 31 & $51.7 \%$ & \multirow{2}{*}{0.657} \\
\hline & NO & 34 & $52.3 \%$ & 29 & $48.3 \%$ & \\
\hline
\end{tabular}

Table 1: Baseline Clinical Characteristics of Study Population

\begin{tabular}{|c|c|c|c|c|c|}
\hline \multirow{2}{*}{} & \multicolumn{3}{|c|}{ Thrombus burden } & \\
\cline { 2 - 6 } & \multicolumn{2}{|c|}{ LTB } & \multicolumn{2}{c|}{ HTB } & P-value \\
\cline { 2 - 6 } & Mean & Standard Deviation & Mean & Standard Deviation & \\
\hline MPV & 8.88 & 0.84 & 10.42 & 0.79 & $<0.001$ \\
\hline platelet & 272.18 & 77.92 & 241.57 & 98.91 & 0.056 \\
\hline
\end{tabular}

Table 2: Laboratory Characteristics of Study Population

The receiver operating characteristics curve of MPV for predicting large thrombus burden was presented in Fig. 1. The area under the ROC curve for MPV was 0.911. The cutoff value for the prediction of HTB was 9.75 Fl. A MPV 9.75 fL predicted HTB with $83.3 \%$ sensitivity and $86.2 \%$ specificity.

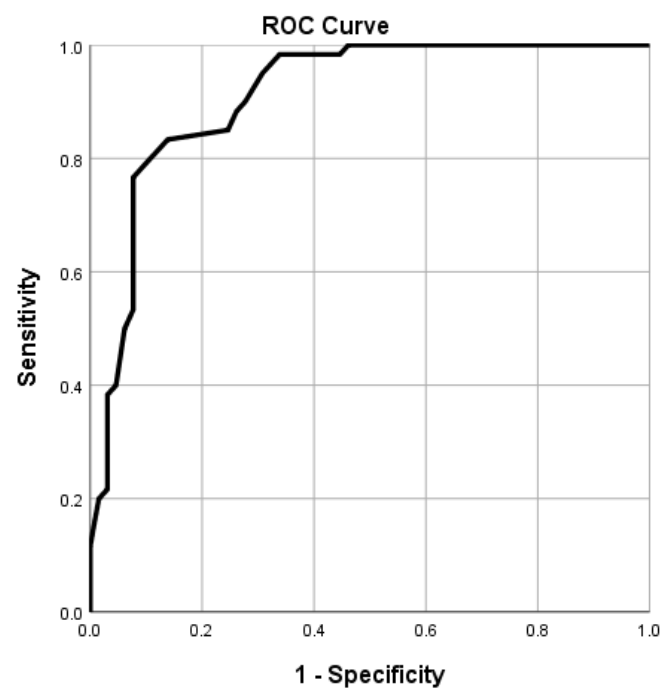

Diagonal segments are produced by ties.

Fig. 1: ROC curve analysis of admission MPV for angiographic large thrombus burden

When we divided patients into two groups based on the MPV cutoff value of $9.75 \mathrm{fL}$ identified in receiver operating characteristics curve analysis, patients with MPV $9.75 \mathrm{fL}$ had a higher prevalence of no-reflow $(6.8 \%$ vs. $0.0 \%$, P 0.047$)$ compared with patients with MPV < 9.75 fL Fig. 2. 


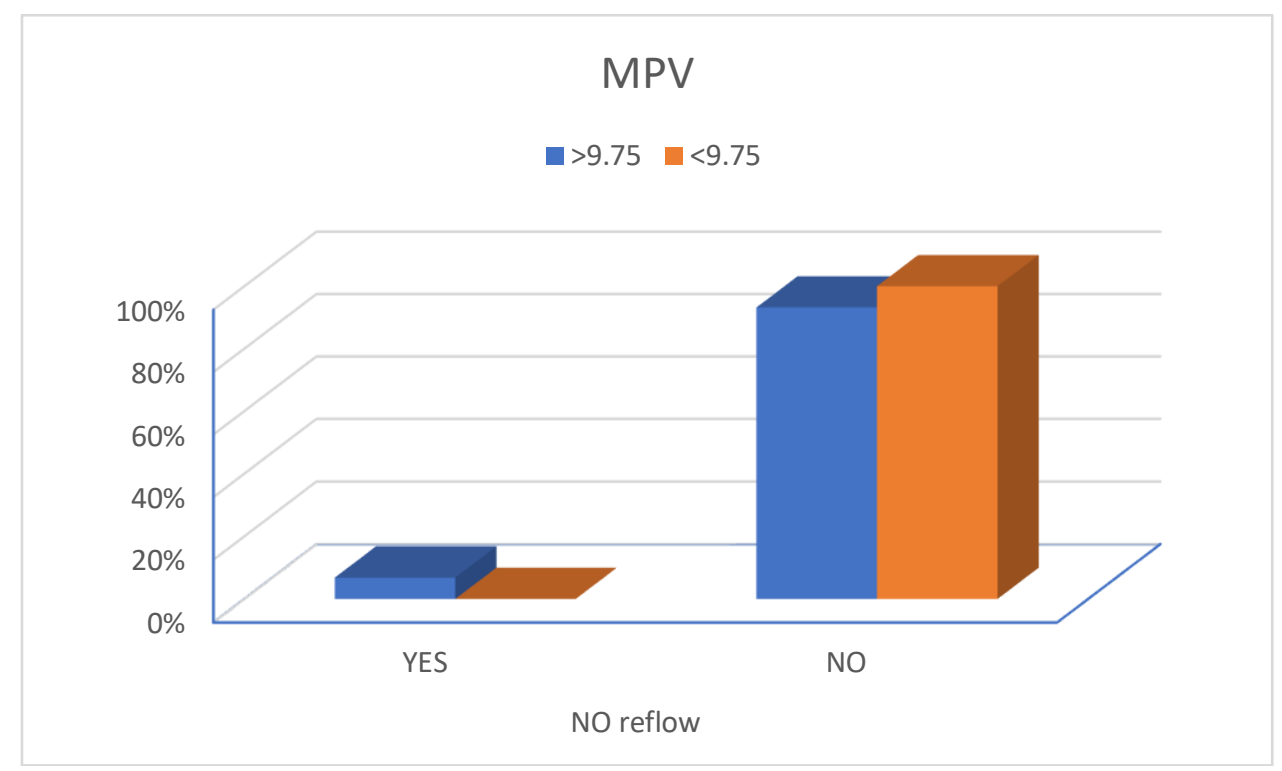

Fig. 2: correlation of MPV and no-reflow of the study population

short-term mortality in patients with STEMI undergoing PPCI, there was a significant difference between MPV

\section{DISCUSSION}

This study was done to demonstrate the usefulness of MPV as a predictor of angiographic thrombus burden in patients who presented with STEMI and underwent PPCI. In this study which was conducted on 125 patients presented with acute ST-segment elevation myocardial infarction who underwent primary PCI, the study sample was divided into two groups: Group 1: patients who had HTB (60 patients). Group 2: Patients who had LTB (65 patients).

Patients in each group were matched to the other group regarding different demographic, clinical, laboratory, and angiographic parameters.

There was no significant difference between the two groups regarding age $(\mathrm{P}$ value $=0.661)$ and sex $(\mathrm{P}$ value $=0.716)$ and most of the patients were males $(109$ patients) $87.2 \%$. The mean age was $59.88 \pm 10.46$. Similarly in the study conducted with Lai et al. ${ }^{7}$ There was no significant difference between age and sex in relation to thrombus burden grade. this study was in concordant with Napodano $\boldsymbol{e t}$ al. ${ }^{8}$ which showed no significant difference between diabetes mellitus and systemic hypertension with thrombus burden grade in STEMI patients. Also, there was no significant difference between the studied groups regarding Killip class. Similarly in the study conducted by Huczek $\boldsymbol{e t}$ al. ${ }^{\mathbf{9}}$ on 398 patients to assess the prognostic value of mean platelet volume (MPV) for angiographic reperfusion and six-month mortality in patients with acute ST-segment elevation myocardial infarction (STEMI) treated with the primary percutaneous coronary intervention (PCI), there was no significant difference between the studied groups as regarding KILLIP class.

With regards to mean platelet volume: in this study, we found that Group 1 that had a high thrombus burden has higher mean platelet volume with a mean of $10.42 \pm 0.79$ compared to group 2 that had a low thrombus burden has lower mean platelet volume with a mean of $8.88 \pm 0.84$. There was a statistically significant difference between the studied groups regarding mean platelet volume. (P-value $<0.001)$. Similarly in the study conducted with Lai et al. $^{7}$ on 649 patients to evaluate the impact of mean platelet volume (MPV) on the intracoronary thrombus burden and with thrombus burden grade in STEMI patients undergoing primary PCI, The admission MPV was significantly higher in the HTB group than in the LTB group $(10.77 \pm 1.22$ vs. $9.95 \pm 1.03, \mathrm{P}<0.001)$. Similarly in the study conducted with Nozari et al. ${ }^{\mathbf{1 0}}$ on 394 patients to determine the association between modified intracoronary thrombus grade and cardiovascular risk factors and initial laboratory findings in patients undergoing primary percutaneous coronary intervention, there was a statistically significant difference between the studied groups as regarding MPV. (P-value $<0.001$ ).

As regards the initial platelet count: in this study, there was no significant difference between the studied groups as regarding the initial platelet count. In contrast, a study which was conducted by Nozari et al. ${ }^{\mathbf{1 0}}$ on 394 patients to determine the association between modified intracoronary thrombus grade and cardiovascular risk factors and initial laboratory findings in patients undergoing primary percutaneous coronary intervention, there was a statistically significant difference between the studied groups as regarding initial platelet count. ( $\mathrm{P}$-value $<0.004$ ). This study was in concordant with Lai et al. ${ }^{7}$ there was no significant difference between culprit's vessel in relation to thrombus burden grade in patients presented with STEMI undergoing primary PCI. Also in this study, there was a significant correlation between the thrombus burden and lesion location, there was HTB in proximal and distal lesions in contrast to LTB was in mid lesions. (P-value 0.046). Also, a significant correlation was found between the thrombus burden and thrombus aspiration. There was increase thrombus aspiration in patients with HTB in comparison to patients with LTB. (P-value 0.028). Similarly in the study conducted with Tungsubutra $\boldsymbol{e t}$ al. ${ }^{11}$ on 202 patients to determine the association between intracoronary thrombus grade and thrombus aspiration in patients undergoing primary percutaneous coronary intervention, there was a statistically significant difference between the studied groups. (P-value <0.002).

As regards TIMI flow; a significant correlation was found between the thrombus burden and initial TIMI flow; there was TIMI flow $<3$ in patients with HTB more than LTB. $(\mathrm{P}$-value $=0.021)$. There was no significant correlation found between the thrombus burden and the final TIMI 
flow. Similarly in the study conducted with Napodano et $a .^{8}$ on 327 patients to determine the association between intracoronary thrombus grade and initial TIMI flow in patients undergoing primary percutaneous coronary intervention, there was a statistically significant difference between the studied groups (P-value $<0.003$ ). There was no significant difference between intracoronary thrombus grade and final TIMI flow in patients undergoing primary percutaneous coronary intervention.

This study was in concordant with Lai et al. ${ }^{7}$ which showed no significant difference between EF with thrombus burden grade in STEMI patients undergoing primary PCI. Also, there was no significant correlation between the study groups with MACCE was observed in terms of Death, CHB, VF, stroke, CIN, and non- fatal CHF. Similarly in the study conducted with Tungsubutra et al. ${ }^{11}$ which showed no significant difference between thrombus burden grade and MACCE in STEMI patients undergoing PPCI.

This study came out with two major concepts with respect to patients who presented with STEMI and underwent PPCI; the first concept is that MPV value on admission was significantly higher in HTB group, the second concept is that MPV is a potential preprocedural independent predictor of thrombus burden in patients with STEMI undergoing primary PCI. Hence, MPV on admission may present a simple preprocedural marker of thrombus burden in patients with STEMI undergoing PPCI, so appropriately selected pharmacological and procedural strategies may be intervened to prevent the incidence of adverse consequences.

\section{CONCLUSION}

In STEMI patients who underwent PPCI, MPV on admission was significantly higher in those who experienced a high thrombus burden.MPV on admission > 9.75 predicted the incidence of HTB with a sensitivity of $83.3 \%$ and specificity of $86.2 \%$.MPV on admission showed an inverse correlation with platelet count. As the MPV increased, the platelet count decreased. This denoted that platelets became hyperaggregable. MPV on admission may serve as a readily available an independent predictor of the thrombus burden in STEMI patients undergoing PPCI.

\section{REFERENCES}

1. Sathananthan J, Watson TJ and Murdoch D. Management of Intracoronary Thrombus. Primary Angioplasty, Springer, 2018; 119-35.

2. Tian C, Song J, He D, Wu J, and Sun Z et al. Predictive value of mean platelet volume/platelet count for prognosis in acute myocardial infarction. Inter Heart J. 2018; 17-212.

3. Koupenova M, Kehrel BE, Corkrey HA, and Freedman JE.Thrombosis and platelets: an update.Eur Heart J, 2016; 38(11): 785-91.

4. Makowski M, Smorag I, Makowska J, Bissinger A andGrycewicz $\mathrm{T}$, et al. Platelet reactivity and mean platelet volume as risk markers of thrombogenesis in atrial fibrillation. Eur Heart J, 2017; 235: 1-5.

5. Kovanen PT, Bot IJE. Mast cells in atherosclerotic cardiovascular disease-Activators and actions. Eur J Pharmacol., 2017; 816: 37-46.
6. Gibson CM, de Lemos J, Murphy S, Marble SJ and McCabe $\mathrm{CH}$ et al. Combination therapy with abciximab reduces angiographically evident thrombus in acute myocardial infarction: a TIMI 14 substudy. Circulation, 2001; 103(21):2550-4.

7. Lai HM, Xu R, Yang YN, Ma YT, and M X et al. Association of mean platelet volume with angiographic thrombus burden and short-term mortality in patients with ST-segment elevation myocardial infarction undergoing primary percutaneous coronary intervention. Catheter Cardiovasc Interv, Am. J. Cardiol .2015; 85(1):72433.

8. Napodano M, Dariol G, Al Mamary AH, Marra MP, and Tarantini $G$ et al. Thrombus burden and myocardial damage during primary percutaneous coronary intervention. Am J Cardiol. 2014; 113(9):1449-56.

9. Huczek Z, Kochman J, Filipiak KJ, Horszczaruk GJ, and Grabowski $\mathrm{M}$ et al. Mean platelet volume on admission predicts impaired reperfusion and longterm mortality in acute myocardial infarction treated with primary percutaneous coronary intervention. $\mathbf{J}$ Am CollCardiol., 2005; 46(2):284-90.

10. Nozari Y, Geraiely B, Kassaian SE, Saroukhani S, and Mortazavi SH. The Association Between Modified Intracoronary Thrombus Grade and Cardiovascular Risk Factors and Initial Laboratory Findings in Patients Undergoing Primary Percutaneous Coronary Intervention. CritPathwCardiol., 2019; 18(3):135-38.

11. Tungsubutra W, Towashiraporn K, Tresukosol D, Chotinaiwattrakul $\mathrm{C}$ andPhankingthongkum $\mathrm{R}$ et al. One-year clinical outcomes of ST-segment elevation myocardial infarction patients treated with emergent percutaneous coronary intervention: the impact of thrombus burden. J Med Assoc Thai., 2014; 97(3):139- 